Special issue of the 2nd International Conference on Computational and Experimental Science and Engineering (ICCESEN 2015)

\title{
Comparison between Real Time PID and 2-DOF PID Controller for 6-DOF Robot Arm
}

\author{
N.G. ADAR* AND R. KOZAN \\ Sakarya University, Mechanical Engineering Department, Sakarya, Turkey
}

\begin{abstract}
Robotics and its applications have become increasingly important in the field of manufacturing industry. Robot manipulators are effectively used for this purpose. Control of manipulators is very important, however, highly nonlinear and multi-input multi-output (MIMO) complex structure of manipulators make the control difficult. This paper presents two-degree of freedom PID controller scheme for a six-degree of freedom rigid robotic manipulator. Traditional PID controllers are widely used due to their simple control structure and ease of implementation in industry. However load disturbances and parametric variation affect the robustness of the controller. The performance of proposed two-degree of freedom PID controller is compared with the traditional PID controllers. Matlab-Simulink program is used for real-time implementation of the proposed method. Experimental results show that two-degree of freedom PID control is better than the traditional PID for manipulator control in real time.
\end{abstract}

DOI: 10.12693/APhysPolA.130.269

PACS/topics: 45.40.LN, 45.80.+R, 07.07.Tw

\section{Introduction}

PID controller is a single loop controller which was introduced in 1940 [1]. Theory of PID control is given in [2]. PID controllers have been extensively used in industrial applications. The success of PID controllers is due to their simple structure and easiness of setting their parameters (Proportional - Integrative - Derivative).

Two-degree of freedom (2-DOF) PID control algorithm is a control method which is a modified form of PID control, proposed to overcome the limitations of PID controllers. 2-DOF PID controller is introduced and its detailed structural information is given in [3-5]. Rakesh et al. [6] have designed 2-DOF nonlinear PID (NPID) controller for a conical tank. They have made a comparison of the NPID and 2-DOF NPID in their application. Sharma et al. [7] have presented a 2-DOF fractional order PID (2-DOF FOPID) controller scheme for a twolink planar rigid robotic manipulator with payload for trajectory tracking task. Simulation results are given in their paper. Boujari et al. [8] have used an active tuned mass damper system, for which the simulations were performed using both PID and 2-DOF PID controller.

In this paper, PID and 2DOF PID controllers are implemented for control of the 6-DOF robotic arm.

\section{Experimental setup}

Robotic arm is an articulated manipulator RRR, with $6 \mathrm{DOF}$, six rotational joints. The robot is mounted on an iron frame. The six joints are the base, shoulder, elbow, wrist, and a gripper, designed to catch and hold work pieces. Dynamixel servomotors were used to control each of these joints. These motors are connected to each other in a daisy chain. The robotic arm setup is shown

\footnotetext{
*corresponding author; e-mail: nadar@sakarya.edu.tr
}

in Fig. 1a. USB2Dynamixel device was used to control the servos from a computer through the serial port.

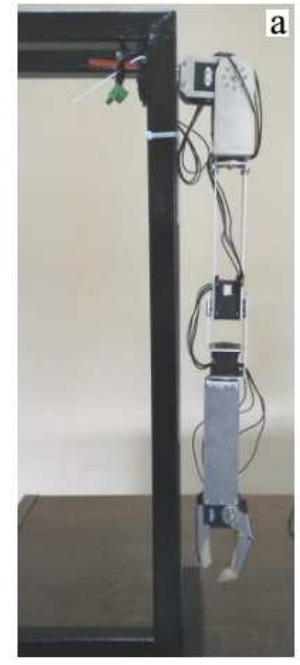

Fig. 1. (a) Experimental Setup. system.

Coordinate system based on Denavit-Hartenberg convention is represented in Fig. 1b. The corresponding parameters of the arm links are shown in Table I.

\section{TABLE I}

Denavit-Hartenberg parameter of the robotic arm.

\begin{tabular}{c|c|c|c|c}
\hline \hline & $d_{i}$ & $\alpha_{i}$ & $a_{i}$ & $\theta_{i}$ \\
\hline 1 & $d_{1}=70 \mathrm{~mm}$ & $90^{\circ}$ & 0 & $\theta_{1}+90^{\circ}$ \\
2 & 0 & $90^{\circ}$ & 0 & $\theta_{2}+90^{\circ}$ \\
3 & $d_{3}=250 \mathrm{~mm}$ & $90^{\circ}$ & 0 & $\theta_{3}+90^{\circ}$ \\
4 & 0 & $90^{\circ}$ & 0 & $\theta_{4}$ \\
5 & $d_{5}=250 \mathrm{~mm}$ & $90^{\circ}$ & 0 & $\theta_{5}$
\end{tabular}


The inverse kinematics of the robotic arm was carried out in [9], where the detailed information is presented.

\section{PID Control and 2-DOF PID control}

The PID algorithm has the following form

$$
u(t)=K_{\mathrm{p}} e(t)+K_{\mathrm{i}} \int e(t) \mathrm{d} t+K_{\mathrm{d}} \frac{\mathrm{d}}{\mathrm{d} t} e(t),
$$

where $u(t)$ is the controller input, $e(t)$ is the control error, which is the difference between the desired response and the actual response and $K_{\mathrm{p}}, K_{\mathrm{i}}$ and $K_{\mathrm{d}}$ are the PID control parameters. The control variable is thus a sum of three parts.

Discrete time PID controller mathematical expression is as follow:

$$
u(t)=K_{\mathrm{p}}+K_{\mathrm{i}} \frac{T_{s}}{2} \frac{z+1}{z-1}+K_{\mathrm{d}} \frac{z-1}{z T_{s}} .
$$

The general form of a 2-DOF PID control system is shown in Fig. 2a and the equivalent block diagram is shown in Fig. 2b. The control system consists of two controllers $C(s)$ and $C_{f}(s)$. It is the combination of a feedforward compensator and a feedback compensator. In this control system, $C(s)$ is the feedback controller while $C_{f}(s)$ is the feedforward controller. The $P(s)$ represents the plant to be controlled by the control system.

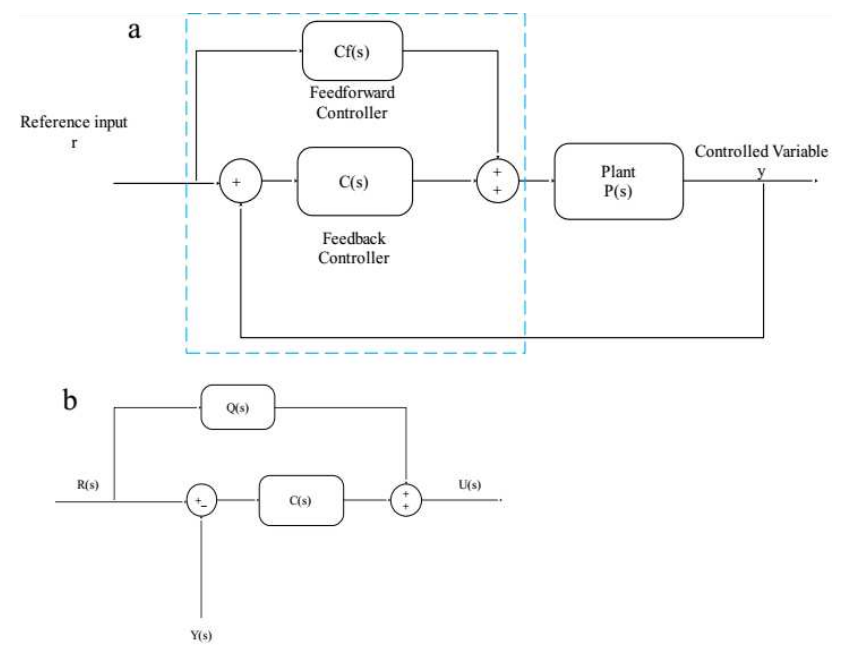

Fig. 2. (a) 2-DOF PID controller block diagram. (b) Equivalent block diagram.

The controller's feedforward section is a PD controller while feedback section is a PID controller.

Transfer functions of $C(s)$ and $C_{f}(s)$ are given below:

$$
\begin{aligned}
& C(s)=K_{\mathrm{p}}\left\{1+\frac{1}{T_{i} s}+T_{\mathrm{d}} D(s)\right\}, \\
& C_{f}(s)=-K_{\mathrm{p}}\left\{\alpha+\beta T_{\mathrm{d}} D(s)\right\},
\end{aligned}
$$

where $\alpha$ and $\beta$ are 2-DOF parameters.

Equivalent 2-DOF PID controller block diagram is shown in Fig. 3.

$$
Q(s)=\frac{[(b-1) P+(c-1) D] s+(b-1) P}{s} .
$$

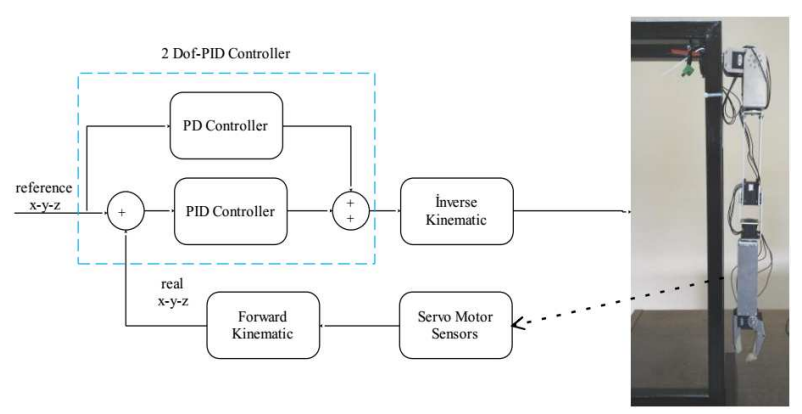

Fig. 3. Block diagram of 2-DOF PID.

\section{Implementation and results}

Current angular position of the each motor can be obtained with sensors integrated into the servo motors. Using this angular positions, the current positions $\left(x_{g}, y_{g}, z_{g}\right)$ are calculated using arm forward kinematics. Error is obtained as the differences between the reference position $\left(x_{r}, y_{r}, z_{r}\right)$ and the real position $\left(x_{g}, y_{g}, z_{g}\right)$.
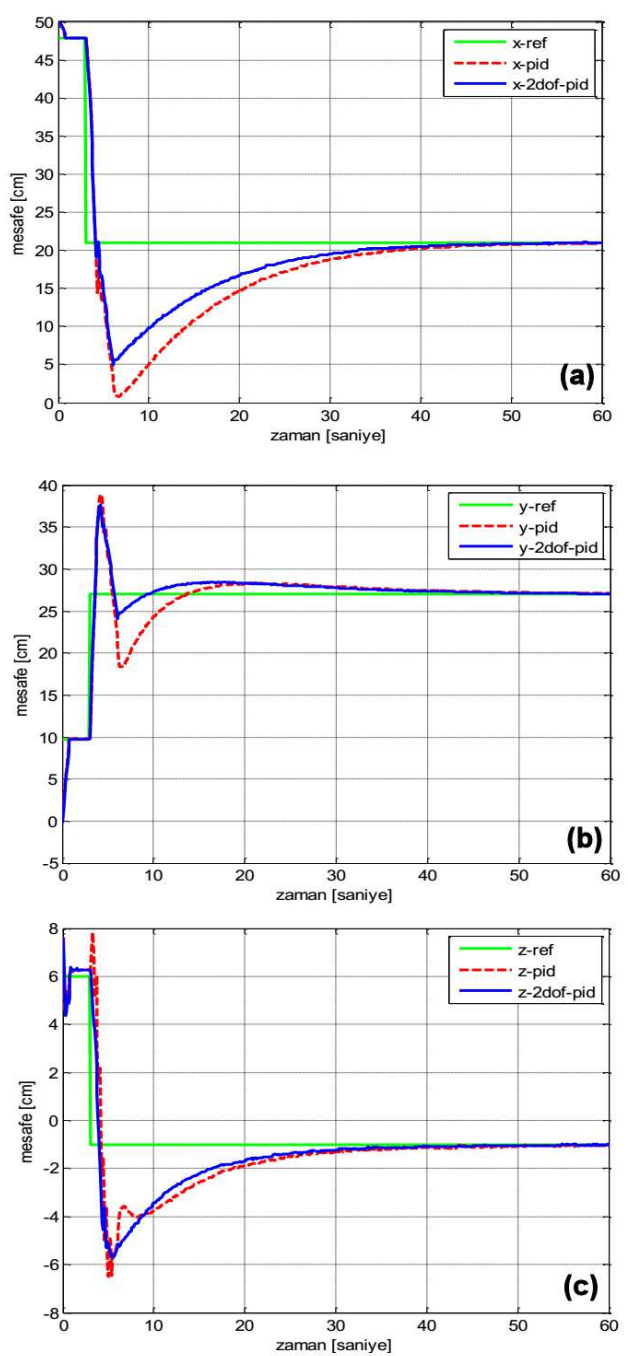

Fig. 4. (a) $x$ position (b) $y$ position (c) $z$ position. Solid line: 2-DOF PID, dashed line: PID. 
This error value is fed into the controller to obtain control signal $u$. This control signal is inserted to the inverse kinematics and the angular positions are computed. These angular positions are sent to each motor to drive them. Algorithm is developed using Matlab/Simulink in both applications. The reference points of the robotic arm are randomly selected. The sampling time was 0.01 seconds. P-I-D coefficients were selected the same for both PID and 2-DOF PID.

Real and reference position, rms and error values were calculated and the results are presented in Table II. Figure 4 shows one of the $(x, y, z)$ positions for both PID and 2-DOF PID controller.

TABLE II

Error, real and reference $x, y, z$ values.

\begin{tabular}{c|c|c|c|c|c|c|c}
\hline \hline & Reference & PID & $\begin{array}{c}\text { PID } \\
{[\%] \text { error }}\end{array}$ & 2-DOF PID & $\begin{array}{c}\text { 2-DOF PID } \\
{[\%] \text { error }}\end{array}$ & $\begin{array}{c}\text { PID } \\
{[\mathrm{rms}]}\end{array}$ & $\begin{array}{c}\text { 2-DOF PID } \\
{[\mathrm{rms}]}\end{array}$ \\
\hline $\mathrm{x}$ & 15 & 14.8441 & 1.0391 & 15.0143 & -0.0956 & 7.2264 & 4.6097 \\
$\mathrm{z}$ & -12 & -12.0826 & -0.6885 & -12.0612 & -0.5103 & 2.6967 & 1.5323 \\
$\mathrm{x}$ & 15 & 14.7962 & 1.3586 & 14.9271 & 0.4863 & 7.5590 & 5.5740 \\
$\mathrm{y}$ & 31 & 31.0955 & -0.3082 & 31.0801 & -0.2583 & 2.8984 & 2.2624 \\
$\mathrm{z}$ & -6 & -6.0516 & -0.8600 & -6.0032 & -0.0541 & 1.5569 & 1.2404 \\
$\mathrm{x}$ & 21 & 21.1599 & -0.7616 & 21.1465 & -0.6974 & 7.2904 & 4.7358 \\
$\mathrm{y}$ & 24 & 24.0811 & -0.3380 & 24.0910 & -0.3790 & 2.6683 & 1.7066 \\
$\mathrm{z}$ & -3 & -3.0063 & -0.2087 & -3.0042 & -0.1404 & 1.6170 & 1.7103 \\
$\mathrm{x}$ & 21 & 20.9958 & 0.0200 & 21.0336 & -0.1601 & 7.4720 & 5.6731 \\
$\mathrm{y}$ & 27 & 27.0420 & -0.1555 & 27.0209 & -0.0774 & 2.6132 & 2.0371 \\
$\mathrm{z}$ & -1 & -1.0452 & -4.5182 & -1.0031 & -0.3103 & 1.5672 & 1.4482 \\
& \multicolumn{7}{|l}{}
\end{tabular}

\section{Conclusions}

This paper presents the performance of PID and 2-DOF PID controllers, which are designed for the control of the robotic arm. It was observed that $x, y$ position of 2-DOF PID has less overshot and faster settling time than that of PID. Even though $z$ position has limited movement in both controllers, it has less vibrations with the 2-DOF PID controller. 2-DOF PID has smaller \% error and the total (rms) error. As a result, it is seen that 2-DOF PID is more effective than the classical PID controller.

\section{Acknowledgments}

This work was supported by Research Fund of the Sakarya University. Project Number: 2013-01-06-032.

\section{References}

[1] M. Babb, Control Eng. 37, 20 (1990).

[2] K.J. Aström, T. Hagglund, PID Controllers: Theory, Design, and Tuning, 2nd ed., Instrument Society of America, 1995.
[3] M. Araki, H. Taguchi, Int. J. Control Automation Syst. 1, 401 (2003).

[4] V.M. Alfaro, R. Vilanova, O. Arrieta, J. Process Contr. 19, 1658 (2009).

[5] V.M. Alfaro, R. Vilanova, O. Arrieta, IFAC Proceedings Volumes 42, 721 (2009).

[6] M.K. Rakesh, R. Satheesh, Thirunavukkarasu, IOSR-JEEE 9, 59 (2014).

[7] R. Sharma, P. Gaur, A.P. Mittal, ISA Trans. 58, 279 (2015).

[8] M. Boujary, A.K. Ghorbani-Tanha, M. Rahimian, H. Rahami, in: Proceedings of the 15th World Conference on Earthquake Engineering 24-28 September, 2012, Sociedade Portuguesa de Engenharia Sismica, Lisbon, Portugal 2012, p. 20065.

[9] N.G. Adar, A. Egrisogut Tiryaki, R. Kozan, Acta Phys. Pol. A 128, B-348 (2015). 